\title{
Conservative treatment of TB Spondylitis in Dorsolum- bar and Lumbar spine
}

\author{
Rabindra Lal Pradhan ${ }^{1}$, Bimal Kumar Pandey ${ }^{l}$, Sashmit Sharma ${ }^{l}$, Shishir Lakhey, \\ Rajiv Raj Manadhar ${ }^{1}$, Kiran Prasad Rijal, Tanup Prasai ${ }^{1}$.
}

${ }^{1}$ Department of Orthopaedic Surgery, Kathmandu Medical College Teaching Hospital,Sinamangal, Kathmandu, Nepal.

\begin{abstract}
Introduction: Tuberculosis (TB) is endemic in South Asia including Nepal. TB spine is the most common musculoskeletal manifestations and can have devastating complications. Early diagnosis by proper history, physical and radiological examination is mandatory. Chemotherapy is effective in most cases while some require surgical treatment.
\end{abstract}

Methods: Forty-four patients with clinico-radiological evidence of TB were treated with four drug regime for 18 months and all patients were followed up till the end of the chemotherapy.

Results: All 44 patients (M-28/ F-16) had back pain not responding to analgesics and physiotherapy while few had constitutional symptoms. Localized tenderness and paravertebral muscle spasm was present in 32/44 (72.7\%) and the rest had palpable swelling at the dorsolumbar and lumbar regions. According to the classification by Oguz et al. the majority of the patients were in the Type I B. Six patients who did not show any improvement in pain and resolution of swelling were admitted and two more drugs (Steptomycin and Ofloxacin) were added.

Conclusions: Conservative treatment of TB spondylitis of the lumbar spine can be treated with chemotherapy and appropriate investigations should be performed in patients with back pain who do not respond to rest, analgesics and physiotherapy.

Keywords: Tuberculosis, thoraco-lumbar spine, chemotherapy

\section{INTRODUCTION}

The incidence of tuberculosis is 63 per 10000 in Nepal according to a recent WHO report. Extra pulmonary tuberculosis (TB) comprised of $14 \%$ of reported cases and bone and joint lesions being one of the most common. ${ }^{1}$ Among them spinal TB accounts for more than fifty percent of cases and can present with nonspecific back pain to paraplegia in our country's situation. Many a time patients present with abscesses, sinus and some deformity and weakness while the average duration of symptoms at diagnosis is around 3-4 months. Early diagnosis and adequate treatment can prevent the devastating complications of neurological deficit and deformity. The dorsal and the dorsolumbar junction is the most common site of infection. Although it has been said that a trail of anti- tubercular drugs (ATT) are justified in a place where $\mathrm{TB}$ is endemic and can be given empirically ${ }^{2,3}$, we believe in obtaining a tissue diagnosis before any ATT if the diagnosis is in doubt and radiological parameters do not show 'typical' lesions. CT guided aspiration and biopsies have been a commonly practiced tool and have the accuracy of $90 \%{ }^{4}$ In many cases where the abscesses are superficial, ultrasound guided aspiration and histology help us in getting a proper diagnosis ${ }^{5}$. It is said that TB spine is a medical disease and should be treated with the same drugs as pulmonary TB, but the response of the drugs may not be the same and many authors have reported on using other regimes of ATT 5,6,7,8,9. The "closed lesions" in spinal tuberculosis have far smaller bacterial population and most of the bacilli in spinal tuberculosis replicate slowly or are

\section{Correspondence}

Dr. Rabindra Lal Pradhan

Department of Orthopaedic Surgery,Kathmandu Medical College Teaching Hospital,Sinamangal, Kathmandu, Nepal.

Email: rabi.ortho@gmail.com 
Table 1.GATA classification of spinal tuberculosis ${ }^{12}$

\begin{tabular}{|l|l|}
\hline Type & Description \\
\hline Type I A & $\begin{array}{l}\text { The lesion located in vertebra one level disc degeneration, no collapse, no abscess, no } \\
\text { neurological deficit. }\end{array}$ \\
\hline Type I B & Abscess formation one or two level disc degeneration, no collapse, no neurological deficit. \\
\hline Type II & $\begin{array}{l}\text { vertebral collapse (pathological fracture) abscess formation, kyphosis (correctable with anterior } \\
\text { surgery), stable deformity with or without neurological deficit sagittal index }<20^{\circ}\end{array}$ \\
\hline Type III & $\begin{array}{l}\text { Severe vertebral collapse, abscess formation, severe kyphosis, instable deformity, with or } \\
\text { without neurological deficit sagittal index } \geq 20^{\circ}\end{array}$ \\
\hline
\end{tabular}

almost dormant. Dormant bacilli tend to retain viability despite chemotherapy, and the need to kill them is the reason why spinal tuberculosis requires a long duration of drug therapy ${ }^{10}$.

Thus, the purpose of this study is to report our experiences in the conservative management of TB spine with chemotherapy for 18 months.

\section{METHODS}

This study was conducted at Kathmandu Medical College teaching hospital (KMCTH) from February 2007 to January 2011. There were a total of 44 patients and all patients presented with mid and lower back pain that was unresponsive to analgesics, rest and physiotherapy. Ambulatory patients were investigated and treated in an outpatient basis and patient with severe pain and bed ridden patients were admitted. Routine laboratory investigations like complete blood count, erythrocyte sedimentation rate (ESR), tuberculin test, liver and renal function tests were performed in all patients. The diagnosis of TB spine was done based on clinical findings, radiological tests like X-rays, and $\mathrm{MR} / \mathrm{CT}$ images and in some cases, aspirations (USG/ CT guided) and open biopsy in two cases. In patients who did not have a tissue diagnosis, all patients had destructive lesions in plain radiographs or CT scans and abscess formation seen in MRI (Figure 1,2). All patients received the standard four drug regime consisting of rifampicin $(15 \mathrm{mg} / \mathrm{kg}$; maximum $600 \mathrm{mg} /$ day), isoniazid (5 mg/kg in adults, maximum $300 \mathrm{mg} /$ day), ethambutol (15-25 mg/kg; maximum $2 \mathrm{~g} /$ day), and pyrazinamide (15-30 mg/kg; maximum $2 \mathrm{~g} /$ day) for three months (HRZE) followed by HRE for three months and then HE for another 11 months totaling ATT for 18 months ${ }^{11}$. Pyridoxine was given to all patients daily till the completion of ATT. All patients were classified according to the GATA classification proposed by Oguz et al. ${ }^{12}$ The decrease in pain and progressive normalization of ESR, bony fusion in plain radiographs and increase in body weight established our diagnosis ${ }^{1}$. Patients who were admitted to the hospital were advised bed rest till the pain subsided and ambulatory patients were advised for some form of brace for a period of three months. Patients with cervical and cranio-cervical, dorsal and sacral TB and patients who underwent anterior or posterior surgical instrumentation were excluded from the study.

\section{RESULTS}

There were a total of 54 patients with TB spine but we could follow 44 patients only who had completed their full course of ATT. Hence our study included 44 patients (M-28/ F-16) with the average of 58.6 years and ranging from 14 to 78 years. All patients had back pain not responding to analgesics and physiotherapy while few had constitutional symptoms. The average duration of symptoms were 3 months and majority of the patients had had multiple consultations and treatment with various drugs and six patients were already on ATT from other centers. Localized tenderness and paravertebral muscle spasm was present in $32 / 44(72.7 \%)$ and the rest had palpable swelling at the dorsolumbar and lumbar regions. Twelve of the patients were admitted to our hospital and all had some form of procedures like ultrasonic guided aspiration of the superficial abscess or biopsy or the patients were too sick. Majority of the patients were neurologically intact while two had partial weakness but none were paraplegic. According to the classification by Oguz et al. ${ }^{12}$ the majority of the patients were in the Type I B. (Table I). Type III lesions were excluded from the study as most of them need some form of surgical intervention. Two patients had skip lesions and one had concomitant lesions in the cervical spine. All patient received the four drug regime with HRZE and majority $38 / 44(86.36 \%)$ and responded in the first six weeks. Six patients who did not show any improvement in 


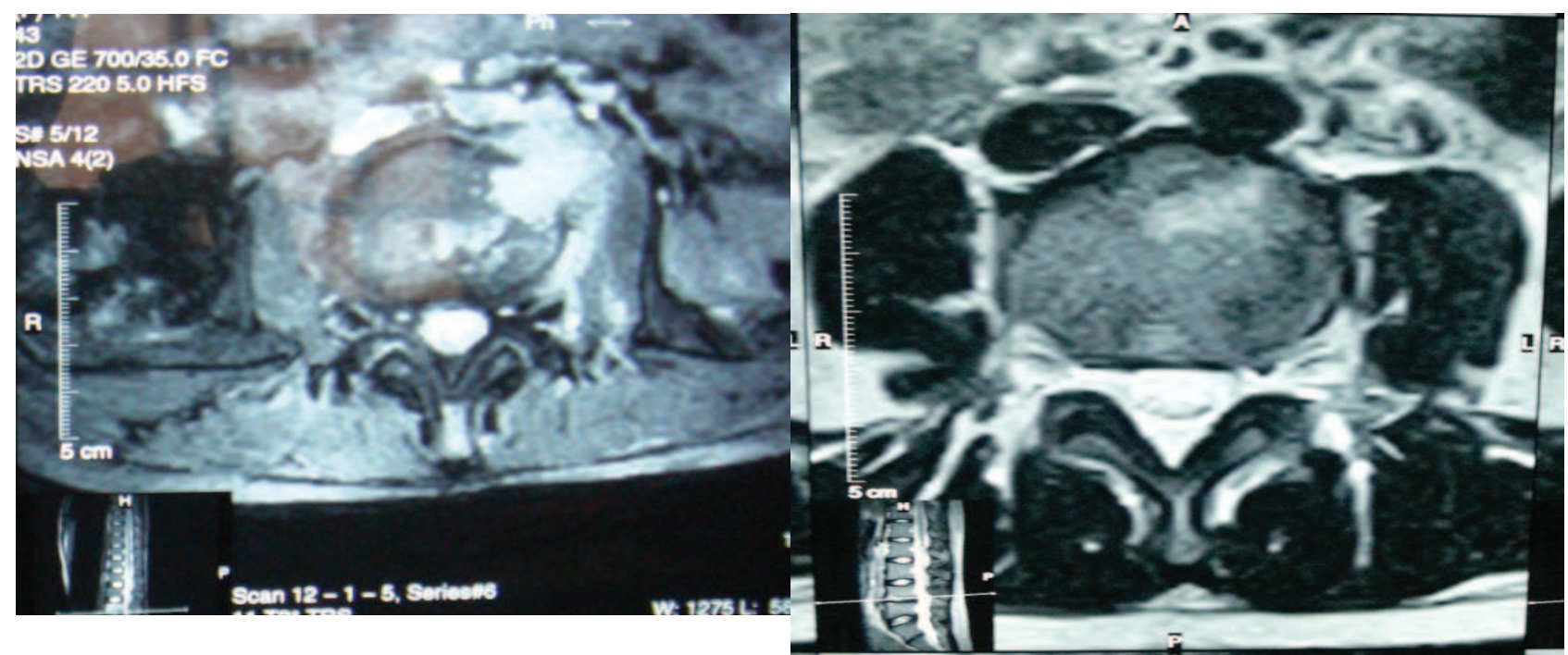

Figure 1 A, B.TB spondylitis in a 18 year old before treatment and after 18 months of ATT

pain and resolution of swelling were admitted and two more drugs (Steptomycin and ofloxacin) were added. Five patients responded with resolution of symptoms in another six weeks but one patient did not respond. This patient underwent immunomodulation therapy as advised by Arora et al. ${ }^{13}$ This patient finally responded after three months of ATT and three episodes of aspiration of the superficial lumbar abscess. Six patients underwent USG guided aspiration of the superficial abscess and two underwent open drainage of their psoas abscess. Among the patients, four developed allergic to rifampicin and five developed derangement of liver function test and other drugs were adjusted by the infectious disease/chest physician. At the end of 18 months of ATT 38 patients had returned to their previous level of activity and had no pain during activities of daily living. Five patients had pain on and off and had to change their occupation and were not able to continue with their farming occupation. One patient still had some residual abscess at the end of 18 months of ATT in MRI but another six months of HR therapy relieved her symptoms and abscess. We did not find any correlation with the amount of abscess formation seen in the MRI and the recovery of the patient.

\section{DISCUSSION}

TB spondylitis is the most common form of bone and joint tuberculosis and also the most debilitating because of bone destruction, deformity and sometimes paraplegia. Initially the lesion starts in the anterior aspect of the body and spreads to the body ${ }^{14,15}$. Paradiscal, anterior and central type of lesions are the most commonly $\operatorname{seen}^{16,17}$.(Fig $\left.1 \mathrm{~A}, \mathrm{~B}\right)$ In our series, among the patients who could afford MRI scan, we had
$68 \%$ as paradiscal followed by anterior and central. In one patient with skip lesion there was a paradiscal lesion in D12/L1 and anterior lesion in L3 vertebrae. (Fig 2)

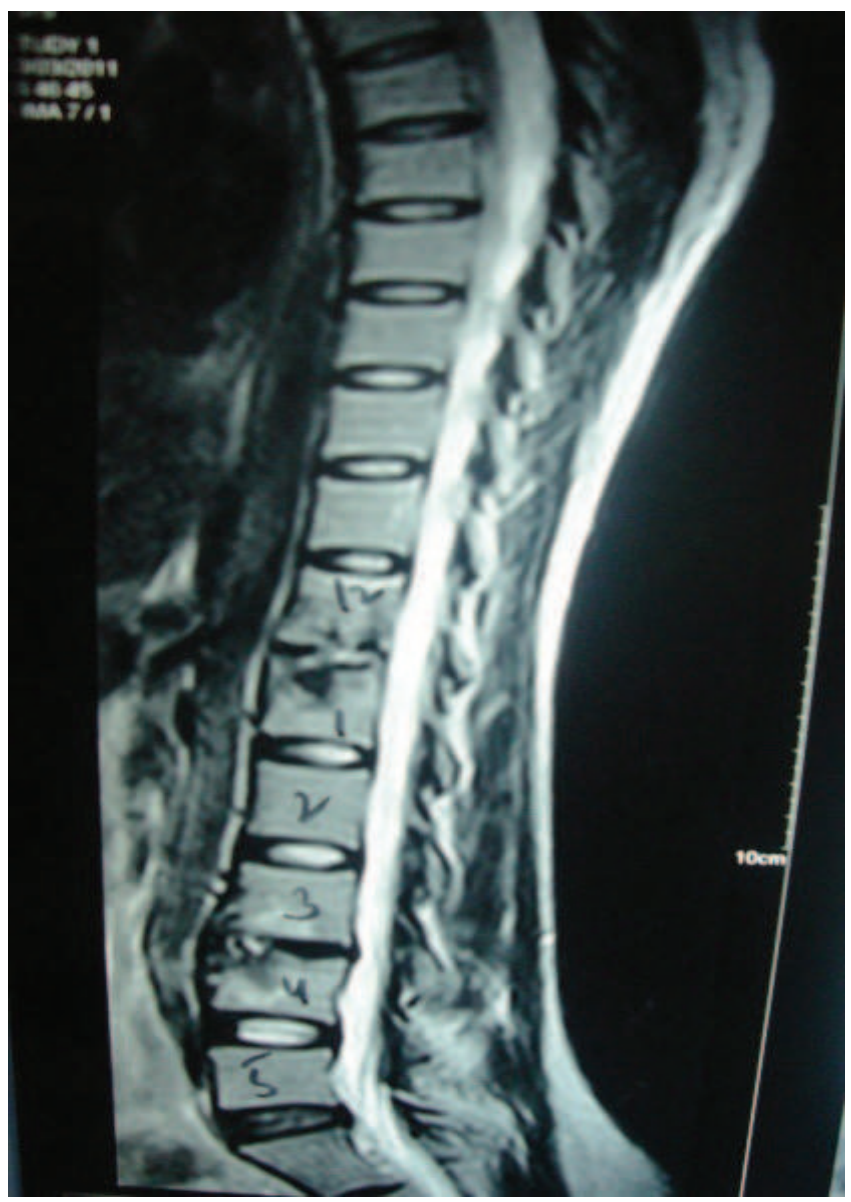

Figure 2. Skip lesions of TB spondylitis

Proper initiation of ATT in the early phases of the disease 
is the key to eradication of the disease and minimizing complications, which can be devastating. All our patients had continuous back pain non- responding to the usual treatment of analgesics and physiotherapy. It has been said that delay in spinal tuberculosis is the rule rather than exception ${ }^{14}$. Hence, proper evaluation of the symptoms and subtle changes in plain X-rays should alert physicians to investigate further in the line of TB spine in unresponsive patients of back and neck pain. Constitutional symptoms such as low fever, malaise, and weight loss did not contribute to a diagnosis in most patients in our series. Radiographic changes associated with TB spine include rarefaction of the vertebral endplates, disc space narrowing, anterior wedging, and bone destruction, but these findings may not be visible on plain radiographs for up to 8 weeks 5 . All others had empirical ATT and all improved in their symptoms with four to six weeks of the drugs. Nevertheless, spinal tuberculosis progresses slowly and insidiously, and early diagnosis before abscess formation and disc degeneration is difficult ${ }^{18}$. For this reason, a detailed patient history is very important in these cases. In the early stages, single-level disc degeneration can be detected by MRI. MRI has been the most sensitive test to diagnose TB spondylitis ${ }^{19-23}$ and in our series where $68 \%$ had a MRI scan and only six patients underwent needle aspiration for histological tests. Although, CTcontrolled biopsy from the destroyed area in the centre of the vertebral body is the gold standard technique for early histopathological diagnosis of these patients, only seven patients underwent the procedure because most could not afford it in our context. There have been many studies that report that in South Asian countries where TB is endemic, histopathogical confirmation is not mandatory and a therapeutic trail of ATT is a practical alternative to taking a biopsy ${ }^{23}$.

The duration and number of drugs to be given in TB spine is still a controversial issue ${ }^{5-9}$. Studies in the past have diverse duration ranging from six months to two years. WHO guideline for extra pulmonary TB has recommended treatment for more than for pulmonary TB but has mentioned for how long ${ }^{1}$. Pulmonary tuberculous lesions are "open lesions," while in contrast, the "closed lesions" in skeletal and spinal tuberculosis do not communicate with air and have far smaller bacterial population. A large part of the bacillary population in pulmonary tuberculosis is rapidly multiplying, whereas most of the bacilli in spinal tuberculosis replicate slowly or are almost dormant. Antitubercular drugs are most effective against the rapidly replicating bacterial population. Dormant bacilli tend to retain viability despite chemotherapy, and the need to kill them (when these start replicating) is the reason why skeletal and spinal tuberculosis require a long duration of drug therapy ${ }^{10}$.

Ataregional workshopheld in Nepal for musculoskeletal TB, participants agreed that drugs should be given for 18 months $^{11}$. Hence we followed the protocol after it was endorsed but had been following the same earlier too. Moon et el ${ }^{18}$ have recommended four drugs for 12 months but we have found the maintenance drugs of rifampicin and isoniazid after seven months have resolved most of the symptoms and abscess in our patients.

The goal of treatment for spinal tuberculosis is to cure the disease with minimum residual spinal deformity. Therefore, treatment at lumbar spine should be aimed at maintenance of lordosis by preventing additional progress of bony destruction during the treatment period and thereafter ${ }^{18}$ The anatomical features of the lumbar and lumbosacral spine make tuberculous infection in this region amenable to conservative treatment as this region can "accommodate" lesions better. A capacious spinal canal, with floating nerve roots which behave like peripheral nerves, can be relatively tolerant to compression because of abscess or granulation tissue, which may develop slowly ${ }^{24}$.

We agree with Bhojraj and Nene ${ }^{24}$ that patients not responding to first line of ATT do not necessarily have to be subjected to surgery. Second line drugs with careful monitoring with consultation with chest physicians can show definite response after some weeks. All paravertebral abscesses do not need to be drained because they may resolve with chemotherapy. Drainage of an abscess may be indicated if it causes symptoms that are simply due to its size and location, such as a flexion deformity of the hip. We had six patients who did not initially respond and more drugs had to be added with repeated aspiration of superficial abscess that were easily accessible by ultrasound guidance. The aspirations improved the general condition of the patient and delayed the rapid progression of the disease. These patients showed definite improvement in symptoms and decrease in the size of the collection in few weeks and none of them had to undergo surgical drainage. Jain et al ${ }^{17}$ have reported that 26 of their 64 patient had neurological improvement with four weeks of ATT alone.

Proponents of earlier surgery argue that surgical debridement and abscess drainage enhance drug treatment but many studies have shown that conservative treatment resolves the lesions and achieve 
NOAJ July-December $2013 \mid$ Vol $3 \mid$ Issue 2

bony healing and union as well, even in patients with partial neurological deficits ${ }^{5,17}$. In our series we have found that conservative treatment for lesions without neurological deficits and cord compression in lumbar spine can be effective in ameliorating the symptoms of TB spine.

\section{CONCLUSIONS}

Management ofTB spondlylitis should beindividualized and antitubercular treatment should be started as early as possible. Although a tissue histological diagnosis is desirable, clinico-radiological diagnosis may be adequate to start ATT medication in suspected cases. Patients who not responding to first line drugs can be started with second line drugs and show response in four to six weeks of initiation of the drugs but needs close monitoring and cooperation with chest physicians. We agree with Tuli that surgery is limited to patients with progressive destruction, improper or slow healing that result in persistent pain, deteriorating neurology, instability and deformity of the spine.

\section{REFERENCES}

1. Treatment of tuberculosis - guidelines WHO 2010

2. Davies PDO. Clinical tuberculosis. Second edition, Chapman and Hall Medical.

3. Jain AK, Kumar S, Tuli SM. Tuberculosis of spine (C1 to D4). Spinal Cord 1999;37:362-369.

4. Abbas A, Rizvi SRH, Mahesri M, Salahuddin HRA. Conservative Management of Spinal Tuberculosis. An initial series from Pakistan. Asian Spine J 2013; 7: 73-80.

5. Kotil K, Alan SM, Bilge T. Medical management of Potts spine in thoracic and lumbar spine: A propective clinical study. J Neurosurg spine 2007; 6:222-228.

6. Yilmaz C, Selek HY, Gürkan I, Erdemli B, Korkusuz Z Anterior instrumentation for the treatment of spinal tuberculosis. J Bone Joint Surg Am. 1999;81(9):1261-1267.

7. Mehta JS, Bhojraj SY Tuberculosis of the thoracic spine. A classification based on the selection of surgical strategies. $J$ Bone Joint Surg Br. 2001;83(6):859-863.

8. Govender $S$, Kumar KP. Cortical allografts in spinal tuberculosis. Int Orthop 2003;27(4):244-8

9. Sundararaj GD, Behera $S$, Ravi V, Venkatesh $K$, Cherian $V M$, Lee $V$. Role of posterior stabilisation in the management of tuberculosis of the dorsal and lumbar spine.2003;85(1):100-6.

10. Tuli SM. Tuberculosis of Spine: A Historical Review Clin Ortho Rel Res 2007; 460: 29-38.

11. Pokharel $R K$ Anti tubercular treatment regime for musculoskeletal tuberculosis. J Nep Med Assoc 2006; 45: 279280.
12. Oguz E, Sehirlioglu A, Altinmakas $M$, et al. A new classification and guide for surgical treatment of spi $\neg$ nal tuberculosis. Int Orthop 2008;32:127-33.

13. Arora A,Nadkarni B, Dev G, Chattopadhya D, Jain A K,Tuli $S M$, Kumar $S$ The use of immunomodulators as an adjunct to antituberculous chemotherapy in nonresponsive patients with osteo-articular tuberculosis J Bone Joint Surg [Br] 2006;88-B:264-9.

14. Bakhsh A. Medical management of spinal tubercu $\neg$ losis: an experience from Pakistan. Spine 2010;35:E787-91.

15. Alothman A, Memish ZA, Awada A, et al. Tubercu $\neg$ lous spondylitis: analysis of 69 cases from Saudi Ara $\neg$ bia. Spine 2001;26:E565-70.

16. Jain AK, Dhammi IK. Tuberculosis of spine: A Review Clin Ortho Rel Res 2007;460: 39-49.

17. Jain AK. Treatment of Tuberculosis of the spine with neurological complications Clin Ortho Rel Res 2002;398:75-84.

18. Moon MS, Moon YW, Moon JL, Kim SS, Sun DH. Conservative Treatment of Tuberculosis of the lumbar and lumbosacral spine Clin Ortho Rel Res 2002;398: 40-49.

19. Liu GC, Chou MS, Tsai TC, Lin SY, Shen YS: MR evaluation of tuberculous spondylitis. Acta Radiol 1993;34:554558 .

20. Desai SS: Early diagnosis of Pott's disease by MRI. J Bone Joint Surg 1994;76:863-867.

21. Lindahl S, Nyman RS, Brismar J, Hugosson C, Lundstedt $C$ : Imaging of tuberculosis. IV. Spinal manifestations in 63 patients. Acta Radiol 1996; 37:506-508.

22. Bala Y, Chandrasekhar VK, Rajesh A, Purohit AK, Rani YJ. Novel magnetic resonance imaging scoring system for diagnosis of spinal tuberculosis: A preliminary report. $J$ Neurosci Rural Pract. 2013;4(2):122-128

23. Rigotti $S$, Zorzi C The importance of early diagnosis with magnetic resonance imaging in spinal tuberculosis. $J \mathrm{Neu}$ rosci Rural Pract. 2013; 4(2): 119.

24. Bhojraj S, Nene A. Lumbar and lumbosacral tuberculous spondylodiscitis in adults J Bone Joint Surg Br 2002;84B:530-534. 